\title{
PROJETO E ANÁLISE DE UMA CLASSE DE ANTENAS REFLETORAS PARA COBERTURA OMNIDIRECIONAL
}

\author{
J. R. Bergmann ${ }^{1}$, F. J. V. Hasselmann ${ }^{1}$ e M. G. C. Branco ${ }^{2}$ \\ 1CETUC-PUC/Rio-Rua Marques de São Vicente 225, Rio de Janeiro 22453-900 \\ 2Fundação CPqD-Rodovia Campinas-Mogi Mirim km.118,5, Campinas, SP 13088-061 \\ bergmann@cetuc.puc-rio.br,flavio@cetuc.puc-rio.br,
}

\begin{abstract}
Resumo - Sugere-se o projeto de uma antena refletora para atender aos requisitos de cobertura omnidirecional em azimute de estações rádio-base para comunicações pontomultiponto na faixa de ondas milimétricas. É utilizada uma superfície refletora cônica de revolução com sua geratriz modelada de forma a produzir um diagrama de radiação em cossecante quadrada no plano vertical para contrabalançar a atenuação de espaço livre na região principal de cobertura. Resultados numéricos ilustram os compromissos envolvidos ao se considerar certos aspectos de projeto e requisitos do alimentador. Explora-se cuidadosamente o papel da Óptica Geométrica e da Óptica Física ao longo dos diversos passos da síntese e análise da antena, objetivando guiar o leitor através dos aspectos primordiais deste estudo em uma sequência apropriada. É também evidenciada a importância de um modelamento adequado do diagrama de radiação do alimentador.
\end{abstract}

Abstract - A single reflector antenna design is suggested for compliance with omnidirectional arimuth coverage requirements in radio base stations for point-to-multipoint services at millimetric bands. Free-space attenuation between transmitter and receiver ends is accounted for by properly shaping the reflector profile to yield a cosecant squared power pattern in the vertical plane. Numerical results illustrate the trade-offs involved when considering certain antenna design aspects as well as feed requirements. The role of both Geometrical and Physical Optics is carefully explored along design and analysis steps with a view towards guiding the reader through key factors of this study in an appropriate sequence. The importance of proper modelling of feed patterns is also evidenced

Palavras-chave: antenas refletoras, cobertura omnidirecional.

\section{INTRODUÇÃO}

Estações rádio-base para comunicações ponto-multiponto utilizam, usualmente, conjuntos de antenas (dipolos, slots, impressas) a certa altura do solo para cobertura omnidirecional, com controle de excitação de elementos para obter uma síntese adequada do diagrama de radiação no plano de elevação. Tipicamente, estes conjuntos apresentam a desvantagem de bandas passantes reduzidas e/ou perdas crescentes com a frequiência devido aos circuitos de controle de fase e potência. Mais recentemente, tem-se voltado a atenção para serviços ponto-multiponto (não necessariamente móveis, mas também fixos) como os sistemas sem fio de banda larga e alta densidade, operando nas faixas de 24 a $38 \mathrm{GHz}$, atualmente em discussão no âmbito da CITEL (Inter-American Telecommunication Commision). Características vantajosas para estes sistemas poderiam incluir a capacidade de reduzir os efeitos de propagação não só pelo emprego de antenas com ganhos elevados mas, também, através de sínteses de diagramas de radiação, na transmissão, que possam compensar a atenuação do sinal de rádio com a distância.

$\mathrm{Na}$ faixa de ondas milimétricas, sistemas compactos de antenas refletoras modeladas podem constituir uma alternativa interessante para os conjuntos usualmente empregados em estações rádio-base devido a sua habilidade em prover diagramas de cobertura customizados, com os requisitos de banda passante sendo satisfeitos pela escolha de alimentadores adequados. Projetos promissores devem, além de produzir diagramas de radiação em cossecante quadrada no plano vertical, para contrapor à atenuação de espaço livre, concentrar a energia radiada abaixo da linha do horizonte visando a redução de interferências. Uma destas configurações, explorada em [1], compreende uma estação nodal com até quatro antenas refletoras para cobertura de quadrantes, cujo esforço de projeto principal foi concentrado no modelamento adequado das superfícies refletoras [2]. Outras propostas têm incluído sistemas duplorefletores compreendendo guias coaxiais ou cornetas cônicas que irradiam campos primários circularmente simétricos e linearmente polarizados sobre um sub-refletor parabólico que, por sua vez, ilumina um refletor cônico modelado de forma a sintetizar o diagrama vertical desejado $[3,4,5]$.

A opção de refletores cônicos é explorada neste trabalho em uma configuração compacta que emprega uma única superfície refletora modelada, circularmente simétrica, alimentada por uma corneta axial conforme ilustrado na Fig. 1. Escolhendo-se apropriadamente os raios das seções circulares concêntricas da corneta, tal que só o modo TEM dominante se propague, obtém-se uma distribuição de campo polarizado axilamente e com independência azimutal, a qual contribui, em conjunto com a forma ponteaguda da superfície refletora, para baixas perdas de retorno. Outros alimentadores, suportando a propagação de modos $\mathrm{TM}_{01}$ ou $\mathrm{TE}_{01}$ para campos linearmente polarizados nos planos horizontal ou vertical, poderiam também ser considerados, embora as principais conclusões deste trabalho, quanto às características da antena explorada, possam ser estendidas a configurações que adotem aqueles projetos alternativos de alimentador. 


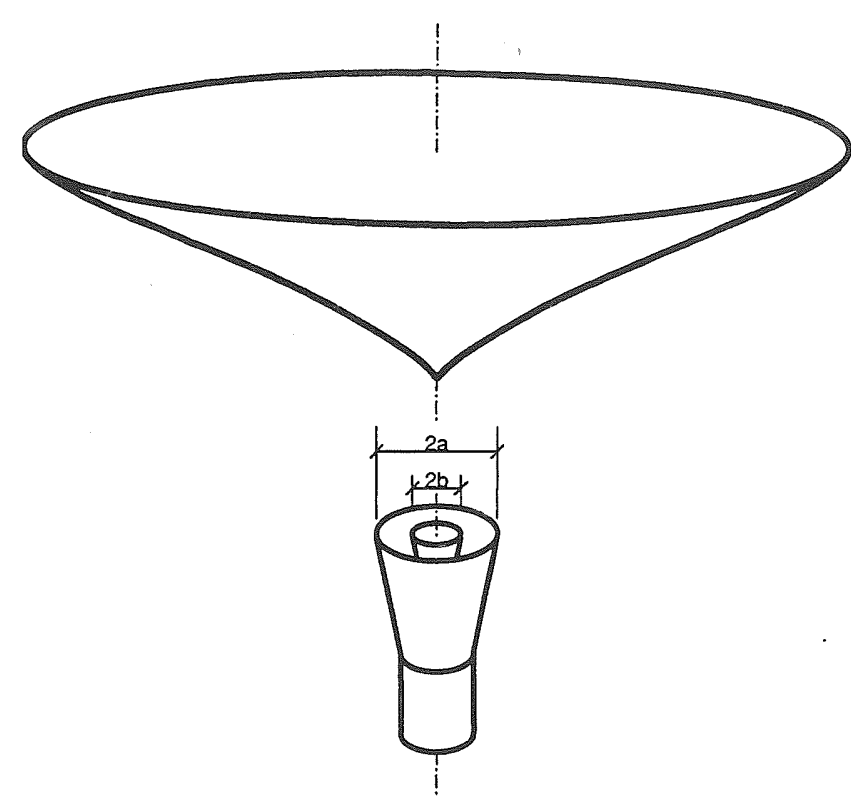

Figura 1. Antena refletora cônica circularmente simétrica alimentado por corneta coaxial.

A geratriz da superfície refletora é sintetizada, inicialmente, de acordo com os postulados da Óptica Geométrica (GO), visando um diagrama secundário de radiação da antena em cossecante quadrada e considerando o modelo simplificado de onda esférica incidente a partir da integração de correntes equivalentes na abertura do alimentador. São então investigadas duas configurações, ambas permitindo as opções de projeto de estruturas de raios refletidos com cáusticas reais ou virtuais. Ou o alimentador é colocado abaixo do refletor e apontado para o zênite, resultando em um lobo principal de transbordamento do refletor acima da linha do horizonte, ou o alimentador aponta para baixo dirigindo a energia de transbordamento para abaixo do horizonte com conseqüente interferência na energia radiada pela estrutura cônica. Desejando-se uma cobertura abaixo do horizonte, com mínima interferência, é também implementado um projeto de corneta com flange corrugada de modo a reduzir sua radiação traseira. Neste estagio, uma análise do campo irradiado pela antena, obtido por integração das correntes induzidas segundo a aproximação da Óptica Física (PO), evidencia a necessidade de um modelamento aprimorado do diagrama do alimentador de modo a prever lobos traseiros mais realistas e evitar discrepância na região principal do diagrama de radiação da antena. Com este fim, conforme sugerido em [6] e já que porções da superfície refletora, próximas ao eixo de simetria, encontram-se na região de campo próximo da corneta, é utilizada uma expansão em ondas esféricas de dados medidos para caracterizar o campo próximo do alimentador

A seguir, é implementado um procedimento de síntese por $\mathrm{PO}$, tendo por solução inicial a superfície refletora sintetizada por $\mathrm{GO}$ e levando em conta o modelo completo do alimentador, de forma a produzir um diagrama de radiação que possa aderir mais suavemente às especificações. Os resultados obtidos são, então, comparados com os obtidos por sínteses por PO mais restritivas quanto aos níveis de lobos secundários em regiões de observação for a da área principal de cobertura.

\section{PROJETO PRELIMINAR}

\subsection{SÍNTESE E CONFIGURAÇÕES DE ANTENA}

As duas configurações básicas da antena e suas estruturas de raios estão ilustradas na Figuras. 2.a e 2.b. No caso da Fig. 2.a, onde o alimentador aponta para o zênite, os esforços, tanto do projeto do alimentador como da síntese do refletor, são direcionados para reduzir a energia de transbordamento acima da linha do horizonte e a interferência causada abaixo daquela linha; pela radiação traseira do alimentador, sobre o diagrama de radiação da antena. Na configuração da Fig. 2.b, enquanto a radiação traseira do alimentador é dirigida para acima da linha do horizonte, a energia de transbordamento é radiada em direção à área de cobertura principal da antena e a existência de uma solução com cáustica de raios refletidos virtual fica limitada a geometrias onde o ângulo de cobertura em campo distante não intercepta o ângulo do cone de raios do alimentador $\left(\alpha_{\max }+\theta_{\max }<90^{\circ}\right)$, evitando, assim, o bloqueio pela superfície refletora; esse bloqueio pode ocorrer também no caso de cáustica real, caso não haja suficiente desobstrução entre os primeiros raios refletidos e os raios incidentes sobre a borda do refletor

Seguindo as considerações acima, implementou-se o procedimento de síntese por GO da superfície refletora através da solução simultânea da lei de reflexão de Snell

$$
\frac{1}{r} \frac{\partial r}{\partial \theta}=\cot a n\left[\frac{\theta-\pi / 2 \mp \alpha(\theta)}{2}\right]
$$

onde os sinais menos e mais correspondem as configurações nas Figs. 2.a e b, respectivamente, $r$ é a distância entre o entro de fase do refletor e os pontos da superfície, $\theta$ denota a direção de incidência dos raios que emergem do centro de fase do alimentador e $\alpha(\theta)$ representa o ângulo entre o raio refletido e a linha do horizonte, e o princípio de conservação de energia

$$
C \int_{\alpha_{1}}^{\alpha} P(\alpha) \sin (\alpha) d \alpha=\int_{0}^{\theta} I(\theta) \sin \theta d \theta
$$

onde $\mathbb{I}(\theta)$ representa o diagrama de radiação do alimentador e $P(\alpha)$ o diagrama de radiação (cossecante quadrada) em campo distante, no plano vertical, especificado para a antena, de modo a fornecer a relação $\alpha(\theta)$ desejada. A constante $\mathrm{C}$ é determinada pela condição que toda a energia interceptada pela superfície seja refletida dentro do range $\left(\alpha_{1}, \alpha_{2}\right)$ e a equação que descreve o perfil do refletor é então obtida inserindo a relação $\alpha(\theta)$ resultante em 


$$
\ln \left(\frac{r}{r_{0}}\right)=\int_{0}^{\theta} \cot a n\left[\frac{\theta-\pi / 2 \mp \alpha(\theta)}{2}\right] d \theta
$$

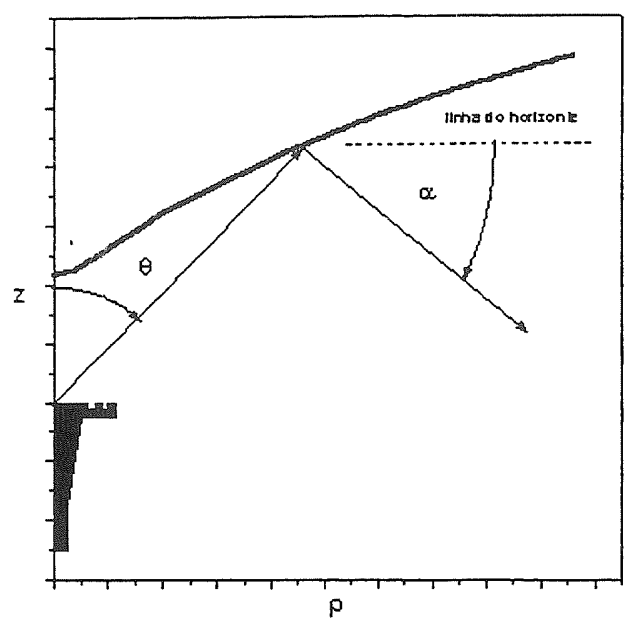

(a)

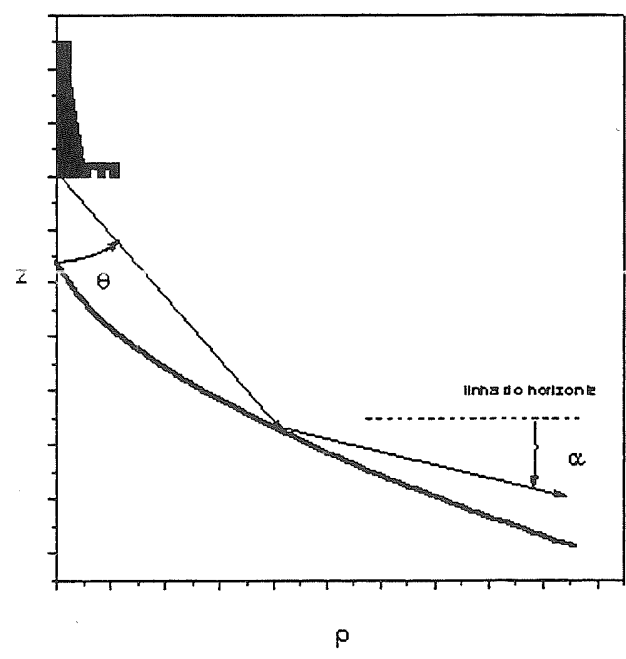

(b)

Figura 2. Configurações possíveis de antena e sua estruturas de raios; (a) alimentador apontando para o zênite, (b) alimentador apontando para baixo.

Ainda, o ângulo $\theta_{\max }$ do cone de raios do alimentador e a distância $\mathrm{r}_{0}$, que define o diâmetro do refletor, são ajustadas para minimizar a energia de transbordamento e reduzir a difração de borda. A superfície refletora fica assim descrita por um conjunto discreto de pontos que é, então, interpolado por uma função global adequada (ver, por exemplo, [2] para uma discussão comparativa das técnicas disponíveis).

\subsection{MODELO SIMPLIFICADO DO ALIMENTADOR}

A corneta coaxial mostrada na Fig. 1 é aqui utilizada devido a sua propriedade de radiação azimutal omnidirecional, conforme revista na Introdução, com o requisito de propagação apenas do modo dominante TEM sendo satisfeito ao se escolher uma diferença (a-b), entre os raios de abertura das seções concêntricas, menor que meio comprimento de onda. Um modelo simplificado, embora útil neste estágio preliminar de projeto, de alimentador consiste em representar o campo incidente no refletor por uma onda esférica com diagrama de radiação obtido pela integração das correntes equivalentes (modo TEM) na abertura da corneta. Obtém-se então, para I $(\theta)$ em (2),

$$
I(\theta)=I_{o}\left[J_{o}(k a \sin \theta)-J_{o}(k b \sin \theta)\right]^{2} / \sin ^{2} \theta
$$

com $\mathrm{k}$ denotando a constante de propagação em espaço livre e $\mathrm{J}_{\mathrm{o}}$ a função de Bessel de primeira espécie e ordem zero. A Fig. 3 os diagramas de radiação do alimentador assim obtidos para $a=0.85 \lambda$ and $b$ variável, correspondentes ao um projeto com um ângulo do cone de raios do alimentador $\theta_{\max }=60^{\circ}$. Observa-se que $b=0.4 \lambda$ provê um bom compromisso entre transbordamento e os níveis de lobos secundários do alimentador que posem interferir no setor principal de cobertura da antena. Para valores maiores de $b$, embora a energia de transbordamento seja reduzida, pode-se obter níveis proibitivos de lobos secundários, o que também ocorre em projetos que utilizem valores maiores de a.

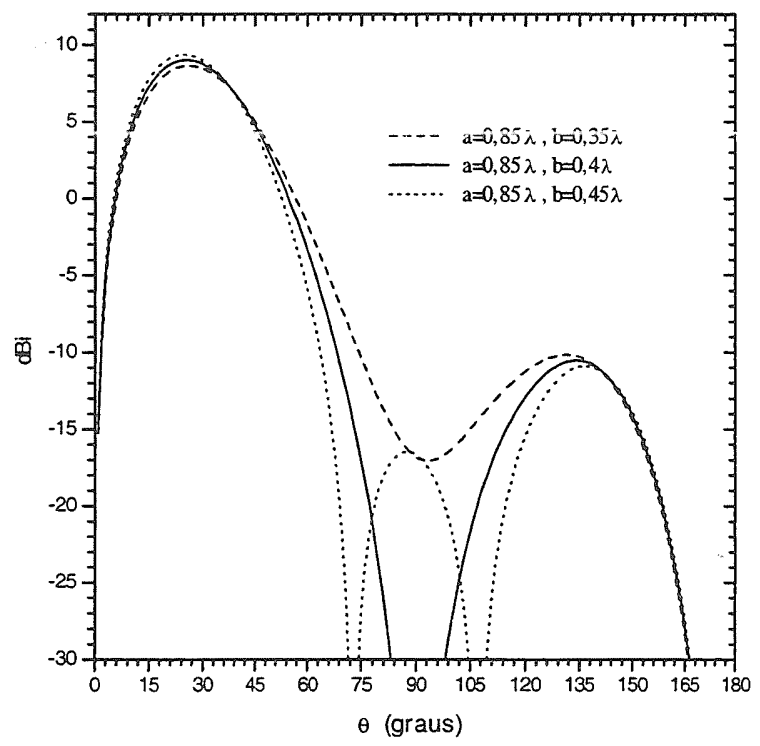

Figura 3. Diagramas de radiação da corneta cônica pelo modelo simplificado de alimentador.

\subsection{RESULTADOS DA SÍNTESE POR GO}

O diagrama de radiação na Fig. 4, obtido através da integração das correntes (PO) induzidas sobre a superfície refletora para a configuração da antena na Fìg. 2.a, é representativa de sínteses por GO com modelos simplificados de alimentador conforme descrito acima. É revelada a influência da energia traseira do alimentador no diagrama de interferência observado para $\alpha>30^{\circ}$, a qual pode, entretanto, ser reduzida pela introdução de uma flange 
corrugada na abertura do alimentador. É também evidenciado na Fig. 4, para a configuração onde o alimentador aponta para o zênite, o papel do radiação traseira da própria antena, para acima da linha do horizonte, o que pode provocar interferências com outros sistemas de comunicações. Embora essas perturbações no desempenho da antena possam ser remediadas através de refinamentos adicionais na síntese, é preciso assegurar-se de que níveis de radiação indesejável precisam ser reduzidos.

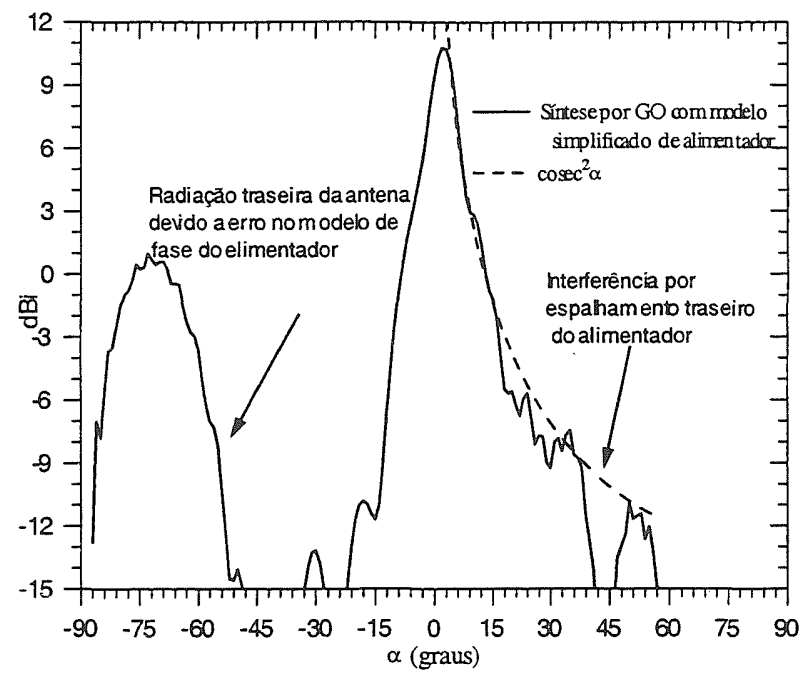

Figura 4. Diagrama de radiação para a síntese por GO da configuração de antena na Fig. 2.a com modelo simplificado de alimentador.

Um exame mais cuidadoso das relações frente-costas obtidas revelou a presença de uma radiação traseira excessiva, ao invés de, conforme esperado de uma análise por $\mathrm{PO}$, um quase cancelamento dos lobos traseiros da antena pela radiação direta do alimentador em oposição de fase. Conforme evidenciado em [6], isto se deveu, quase certamente, ao modelo simplificado utilizado para o diagrama de radiação do alimentador. Tendo isto em mente, as sinteses por $\mathrm{GO}$ foram refeitas considerando um modelo mais adequado de alimentador onde, já que porções da superfície refletora estão próximas da abertura da corneta, as características de campo próximo, assim como os efeitos da flange na abertura, foram levados em conta. Isto foi implementado através de uma expansão em harmônicos esféricos do diagrama medido de um protótipo de alimentador construído com esta finalidade, permitindo obter-se uma representação mais adequada para a distribuição de amplitude do campo sobre a superfície refletora. Uma comparação entre os diagramas de radiação do alimentador, obtidos pela utilização dos modelos simplificado e mais rigoroso, é ilustrada na Fig. 5 para as dimensões $a=0.85 \lambda$ e $b=0.4 \lambda$.

Ainda considerando a configuração de antena na Fig. 2.a e utilizando a representação apropriada, acima descrita, para a energia incidente no refletor, obteve-se o diagrama de radiação mostrado na Fig. 6. Embora os efeitos de espalhamento traseiro estejam agora corretamente acessados, uma deficiência relevante das sínteses por GO está ainda presente. Como os efeitos de difração não são incorporados à formulação de síntese, o diagrama (da PO) resultante apresenta uma distribuição ondulatória que se desvia do decaimento suave especificado pela cossecante quadrada. Obviamente, este efeito só poderia ser contornado através da implementação de uma síntese difrativa.

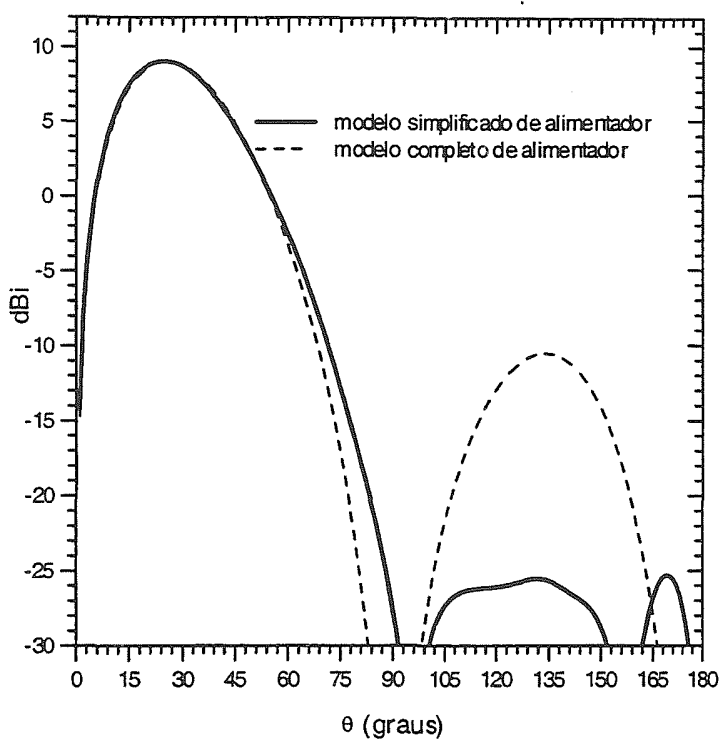

Figura 5. Diagramas de radiação do alimentador pelos modelos simplificado e completo.

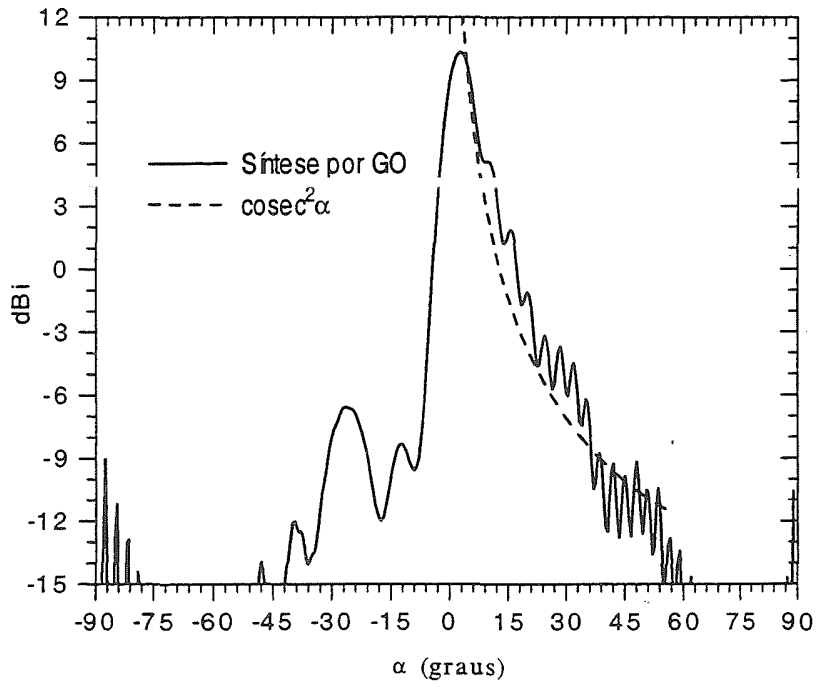

Figura 6. Diagrama de radiação para a síntese por GO da configuração de antena na Fig. 2.a com modelo completo de alimentador.

\section{SÍNTESE DIFRATIVA}

A etapa seguinte de projeto incorpora a síntese por PO da superfície refletora, levando em conta o modelo completo de alimentador, conforme justificado nas Seções anteriores, e utilizando a superfície sintetizada por GO como solução inicial. O procedimento básico envolve a minimização de uma função objetiva descritiva da diferença entre os diagramas de radiação obtido pela análise por $\mathrm{PO} e$ especificado (cossecante quadrada) na região principal de cobertura. A velocidade de convergência do algoritmo para uma tolerância pré-estabelecida é claramente influenciada 
pela capacidade da expansão em série escolhida para acomodar globalmente os conjuntos de pontos resultantes das superfícies sintetizadas e pelo número de seus coeficientes a serem determinados. Estes aspectos, entretanto, excedem o escopo da presente discussão e serão explorados em trabalho futuro.
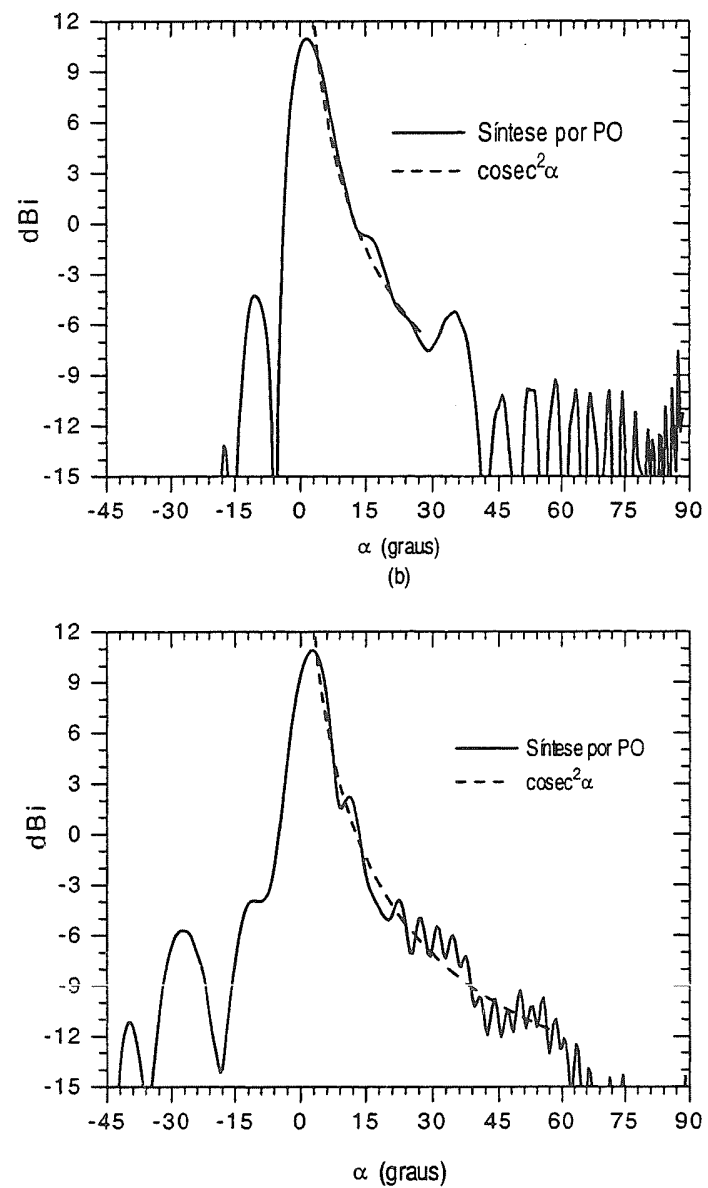

(a)

Figura 7. Diagramas de radiação para as sínteses por PO com modelo completo de alimentador; (a) alimentador apontando para o zênite, (b) alimentador apontando para baixo.

Os diagramas de radiação mostrados nas Figs. 7.a,b correspondem às configurações de antenas nas Figs. 2.a,b com refletores de diâmetro $40 \lambda$ e níveis de iluminação de borda de $-2 \mathrm{dBi}$ e $\mathrm{OdBi}$, correspondendo a ângulos do cone de raios do alimentador $\left(\theta_{\max }\right)$ de $60^{\circ}$ e $55^{\circ}$, respectivamente. $E$ evidenciada, como esperada, uma conformidade mais suave com o decaimento em cossecante quadrada especificado para o lobo principal. Com relação à síntese correspondente à Fig. 7.b, o diagrama foi controlado só até $\alpha=30^{\circ}$ de modo que, já que $\theta_{\max }=55^{\circ}$, a condição $\left(\alpha_{\max }+\theta_{\max }<90^{\circ}\right)$ fosse satisfeita, assim explicando o overshooting e o diagrama de interferência observado para $\alpha>30^{\circ}$; caso o diagrama fosse controlado até valores superiores de $\alpha$, entretanto, o ângulo do cone de raios do alimentador seria coerentemente reduzido, acarretando em níveis mais elevados de iluminação de borda e na possibilidade de uma maior energia de transbordamento. $O$ primeiro lobo secundário que se observa acima do horizonte 106 resulta de uma frente de onda quase plana na abertura da antena, típica em projetos com alto ganho. Com relação à Fig. 7.a, foi utilizado um ângulo maior para o cone de raios do alimentador $\left(\theta_{\max }=60^{\circ}\right)$, com consequiente redução do efeito de transbordamento para $\alpha=-30^{\circ}$. O uso de valores ainda maiores para $\theta_{\max }$ com o fim de reduzir este efeito, resultaria, entretanto, em uma superfície refletora mais alongada verticalmente, com uma pequena abertura efetiva no plano vertical e inerente redução de ganho. Ainda, o diagrama de cobertura principal foi controlado, na síntese correspondente, até $\alpha=60^{\circ}$, tendo em vista os baixos níveis de energia além daquele range; não-obstante, ainda se observa um diagrama de interferência para $\alpha>30^{\circ}$, devido à presença de algum espalhamento traseiro pelo alimentador.
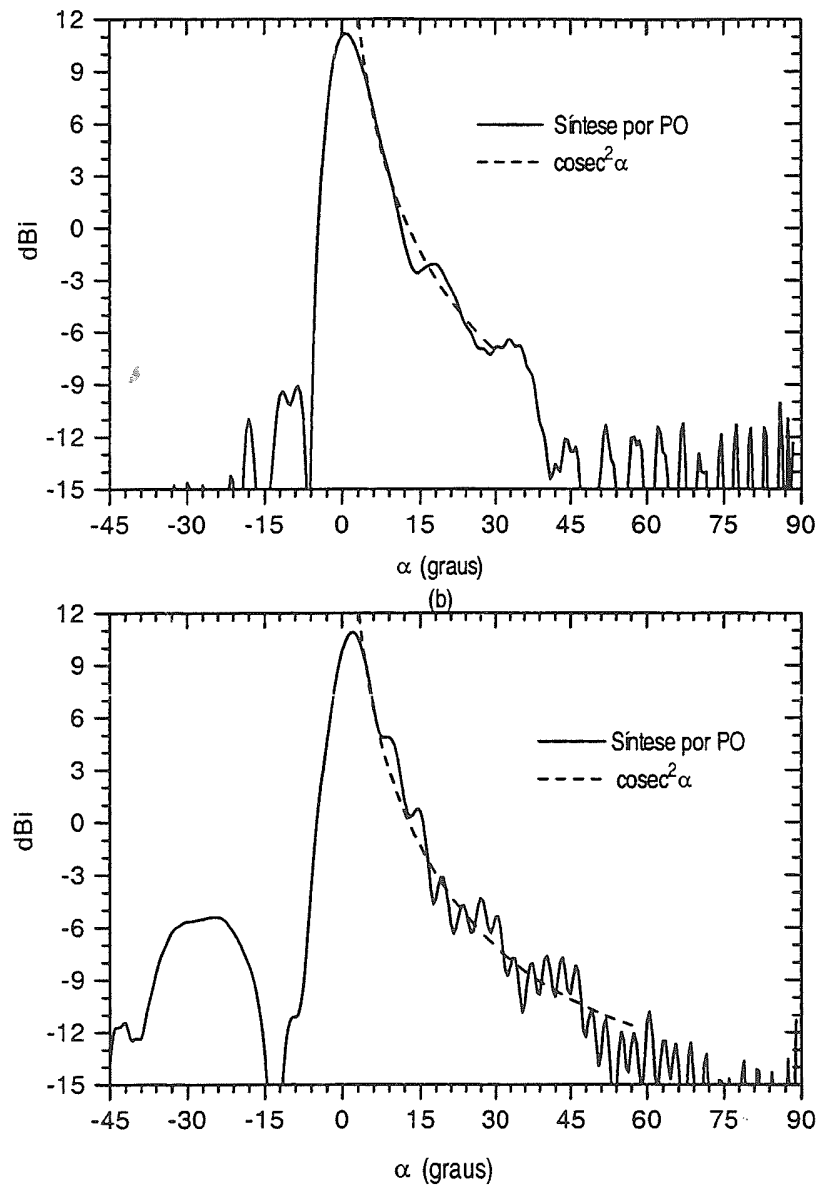

(a)

Figura 8. Diagramas de radiação para as sínteses por PO com modelo completo de alimentador e restrições de lobos secundários; (a) alimentador apontando para o zênite, (b) alimentador apontando para baixo

Os conjuntos de diagramas de radiação apresentados nas Figs. 8.a,b foram obtidos impondo-se restrições quanto aos níveis de lobos secundários em sinteses por PO com as mesmas soluções iniciais (sínteses por GO) utilizadas nos exemplos precedentes. A Fig. 8.b mostra que, além de um nível mais baixo de lobos secundários, foi possível obter alguma redução de transbordamento pela extensão do controle da síntese do diagrama da cobertura principal até $\alpha=35^{\circ}$. Por outro lado, para a confuguração de antena onde o alimentador aponta para o zênite, o diagrama de radiação 
obtido na Fig. 8.a ilustra a dificuldade em se controlar simultaneamente os níveis de lobos secundários e de transbordamento observados em ângulos de observação pequenos acima do horizonte (ver Fig. 7.a) sem perturbar progressivamente o diagrama do lobo principal, face às variações de fase introduzidas nas correntes sobre o refletor. Fica portanto evidenciado que, independentemente de quão criteriosamente sejam conduzidas as diversas etapas da síntese, o projetista deve se deparar com compromissos de desempenho da antena.

Deve-se ainda ressaltar, em relação a sínteses por PO com restrições de lobos secundários, que, por as mesmas não envolverem exercícios de traçado de raios, pode se fazer necessária uma análise adicional para verificar a desobstrução dos raios refletidos pela superfície refletora, já que um maior controle da região de lobos secundários pode resultar em porções da superfície com curvaturas mais acentuadas.

\section{AGRADECIMENTOS}

Este trabalho foi parcialmente suportado pela Telecomunicações Brasileiras S.A. através do contrato PUC-TELEBRÁS P\&D/DRT/775/97-JDPqD e pela Financiadora de Estudos e Projetos através do Convênio FINEP 41.96.0901.00-PRONEX.

\section{REFERÊNCIAS}

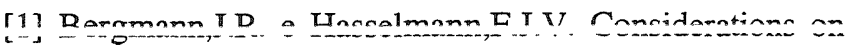
the Design and Analysis of a Shaped Reflector Antenna for Nodal Stations in Metropolitan Areas. Proc. IEE $9^{\text {th }}$ Int. Conf. Antennas Propagat., Vol. 1, pp. 195-198, 1995.

[2] Bergmann,J.R., Hasselmann.F.J.V., Teixeira,F.L. e Rego,C.G. A Comparison between Techniques for Global Surface Interpolation in Shaped Reflector Analysis. IEEE Trans. Antennas Propagat.,Vol. AP-42,no.1,pp.47-53, 1994.

[3] Orifice,M. e Pirinoli,P. A Dual Reflector Antenna for Omnidirectional Coverage. IEEE AP-S Int. Symp. Digest, pp. 274-277, 1993.

[4] Besso,P., Bills,R., Brachat,P. e Vallauri,R. A Millimetric Wave Omnidirectional Antenna with Cosecant Squared Elevation Pattern. Proc. IEE 10 ${ }^{\text {th }}$ Int. Conf. Antennas Propagat., Vol. 1, pp. 448-451, 1997.

[5] Norris,A.P. e Waddoup,W.D. A Millimetric Wave Omnidirectional Antenna with Prescribed Elevation Shaping. Proc. IEE $4^{\text {th }}$ Int. Conf. Antennas Propagat., Vol. 1, pp. 141-145, 1985.

[6] Rahmat-Samii,Y. e Imbriale,W.A. Anomalous Results from $\mathrm{PO}$ Applied to Reflector Antennas: The Importance of Near Field Computations. IEEE AP-S Int. Symp. Digest, pp. 816-819, 1998.

José Ricardo Bergmann obteve o diploma de Engenheiro Eletricista pela Universidade Federal do Rio Grande do Sul em dezembro de 1975. Recebeu o título de Mestre em
Ciências em Engenharia Elétrica pelo Instituto Militar de Engenharia em 1979 e o título de Doctor of Phylosophy pel university of London em 1986. É Coordenador Central de Pós-Graduação e Pesquisa da Pontifícia Universidade Católica do Rio de Janeiro e chairman do Capítulo Conjunto Rio de Janeiro das Sociedades AP/MTT/ED do IEEE. Seus principais interesses em pesquisa são em métodos numéricos aplicados ao eletromagnetismo, síntese e análise de antenas refletoras.

Flávio José Vieira Hasselmann obteve os diplomas de Engenheiro Eletricista (Telecomunicações) e Mestre em Ciências em Engenharia Elétrica pela Pontifícia Universidade Católica do Rio de Janeiro em 1974 e 1976, respectivamente, e de Doctor of Phylosophy (Electrophysics) pelo Polytechnic Institute of New York (hoje, Polytechnic University) em 1980. É coordenador do grupo de pesquisas em antenas do CETUC (Centro de Estudos em Telecomunicações da PUC/Rio), coordenador do Programa de Pós-Graduação em Engenharia Elétrica da PUC/Rio e vice-chairman do Capítulo Conjunto Rio de Janeiro das Sociedades AP/MTT/ED do IEEE. Na SBrT, foi membro do Conselho Deliberativo no período 1983-1985, coordenador técnico do IV Simpósio Brasilleiro de Telecomunicações e editor da área de antenas e propagação da revista da SBrT no período 1987-1991. Seus principais interesses em pesquisa são em métodos assintóticos em eletromagnetismo, antenas refletoras e espalhamento em cobertura de sistemas celulares.

Marcos Guimarães Castello Branco obteve o diploma de Engenheiro Eletrônica pela Universidade Federal do Rio de Janeiro em dezembro de 1979. Recebeu o título de Mestre

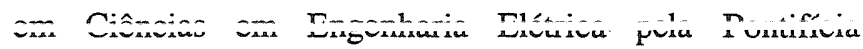
Universidade Católica do Rio de Janeiro em 1982. Atualmente é gerente Divisão de Sistemas Radiantes da Fundação CPqD e atua na CBC-9 da ANATEL/UIT . Seus principais interesses em pesquisa são em métodos numéricos aplicados ao eletromagnetismo, alimentadores e de antenas refletora 